\title{
Design of resonant optical cavities for ultrasound detection using rigorous electromagnetic modelling
}

\author{
Dylan M. Marques*, James A. Guggenheim*, Rehman Ansari*, Edward Zhang*, Paul C. Beard* and Peter R. T. Munro* \\ ${ }^{*}$ Department of Medical Physics and Biomedical Engineering, University College London, London, U.K. \\ Email: dylan.marques.17@ucl.ac.uk
}

\begin{abstract}
Detection of very weak ultrasound waves with a Fabry-Perot interferometer has enabled a new range of biomedical applications such as photoacoustic imaging. We propose a realistic model of the optical readout of this device valid for arbitrary focussed readout beams and optical fibre based detection.
\end{abstract}

Index Terms-Photoacoustic, Electromagnetic modelling, Fabry-Perot interferometer

\section{INTRODUCTION}

Optical systems based on Fabry-Perot interferometers (FPIs) are widely used for ultrasound detection in photoacoustic imaging since they are very sensitive to very weak perturbations in cavity thickness [1]. Its optical performance is typically predicted using a simplified model, which calculates the intensity of light reflected from the FPI assuming plane wave illumination (i.e., an Airy function). However, this approach is not sufficient to model the interrogation of a FPI using nonplanar incident laser beams and optical fibre based readout.

A more accurate model that considers the interrogation beam as a focused laser beam and optical fibre detection has been developed. The FPI is modelled as a multilayer stack and full vectorial description of light is employed (multilayer stack model). A secondary model has been developed that allows us to simulate the setup based only on the mirrors' reflectivity and the respective optical path length inside the FPI's cavity (angular Airy model).

Using a more realistic model we are able to study how interferometer transfer functions (ITFs), i.e. reflected power as a function of wavelength, behave. Also we increase our understanding of the FPI's working principle.

\section{MODELS}

Figure 1 depicts the interrogation system modelled in this paper.

The setup uses a interrogation beam originating from an optical fibre which is then focused into the FPI by a $4 \mathrm{f}$ lens system. After the reflection the light propagates back via the 4f lens system for detection by an optical fibre. Equations 1 and 2 are a mathematical description of the model.

$$
\begin{array}{r}
|\alpha|^{2}=k^{4} 16 \pi^{4}\left|\int_{0}^{\frac{\pi}{2}} \int_{0}^{2 \pi} E_{i}^{2} r(\theta) \cos (\theta) \sin (\theta) d \phi d \theta\right|^{2} \\
E_{i}=\frac{M F D^{2}}{16 \pi^{2}} e^{\frac{-M F D^{2} k^{2} \sin (\theta)^{2}}{4}}
\end{array}
$$

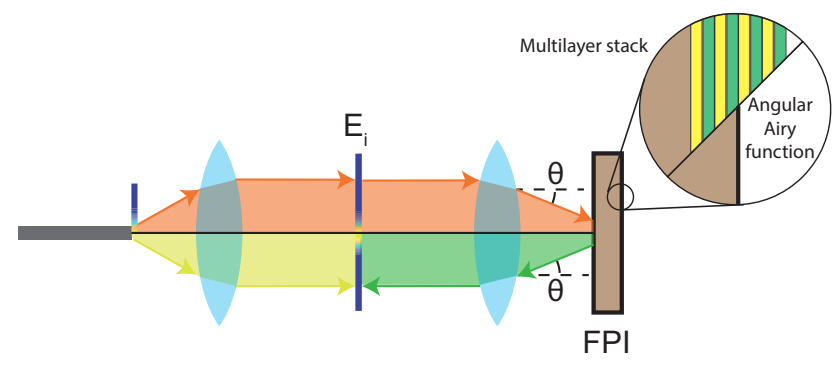

Fig. 1. Models representation. The expanded view represents the two FPI interpretations: as a multilayer stack and as a cavity between infinity thin mirrors (angular Airy model).

where $|\alpha|^{2}$ represents power of light coupled into the detection fibre, and the measured intensity. $k=\frac{2 \pi}{\lambda}$ and $M F D$ is the fibre mode field diameter.

The FPI is modelled in two different ways: using the multilayer matrix formalism [2] and also by the Airy function [3]. Thus, according to the model considered, $r$ has a different meaning.

The latter model (angular Airy function) allows the FPI to be modelled using only the mirrors' reflectivity which can be measured experimentally but the phase changes in the mirrors' transmissions and reflections are neglected.

\section{VALIDATION}

Figure 2 a) shows multiple ITFs and the respective comparison with both newly developed models, pre-existing and experimental measured ITFs.

Comparing the pre-existing Airy model with experimental measurements and the multilayer stack and angular Airy models, a significant difference is that the Airy function's fringes go to zero. This is explained by the fact that the pre-existing model considers the plane wave case and not the focused case. In the newly developed models, a spectrum of plane waves is considered and since each plane wave has slightly different resonance wavelength, there is a decrease in the ITF visibility.

The angular Airy function and multilayer stack models have a different resonance wavelength because in the angular Airy model, the resonance wavelength depends only on the optical path length inside the cavity, whereas in the multilayer stack model the resonance wavelength is also depend on the 


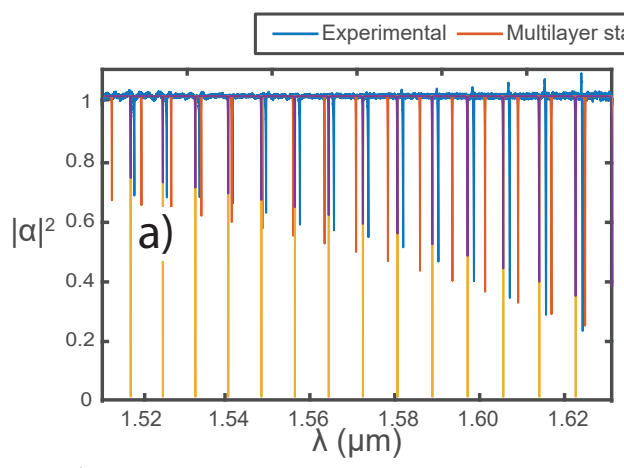

Airy function —Angular Airy function
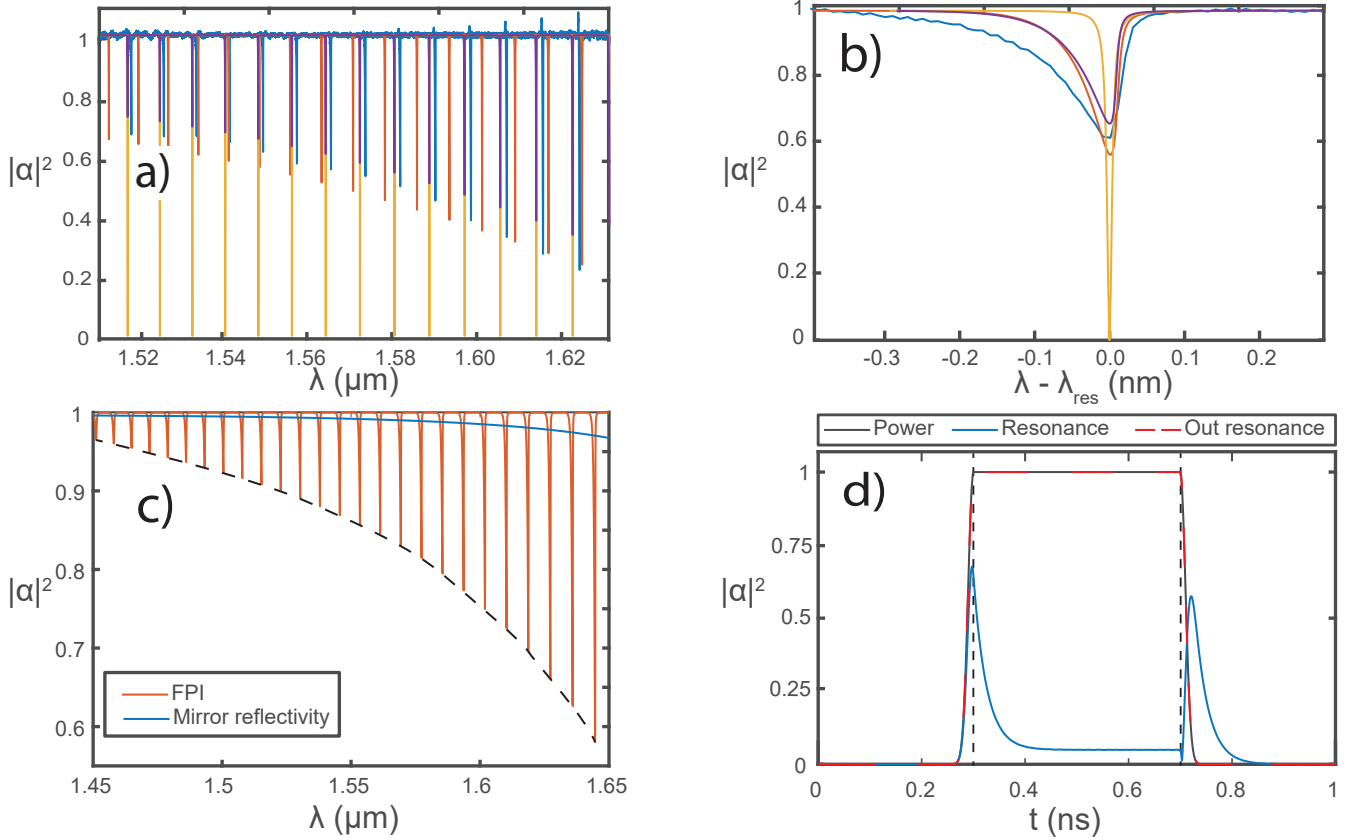

Fig. 2. Experimental and modelled results. a-b) Experimental and simulated data from the developed and pre-existing models. A focus spot of $46 \mu \mathrm{m}$ was considered. b) represents the $1547 \mathrm{~nm}$ to $1549 \mathrm{~nm}$ fringes centered in the respective resonance wavelength. c) Modelled data considering the multilayer stack model with the respective mirrors' reflectivity. The dashed line represents the envelope. d) Modelled time response of a FPI in resonance and out of resonance with the respective power curve. The plane wave case is considered in this graph and the FPI is interpreted as a multilayer stack. The electric field time dependence is omitted for display purposes.

phase changes on reflection at the mirrors. This last point also explains the difference in the fringes periodicity between models.

Both newly developed models and the measured data show the fringes' asymmetry and visibility variations characteristics of the focused case. However the experimental resonant positions and periodicity are slightly different than modelled due to small optical path mismatch inside the cavity. Also the experimental data has chromatic aberrations at wavelengths far from $1550 \mathrm{~nm}$ which aren't modelled.

\section{VISIBILITY VARIATIONS}

When an ITF is measured using a focused Gaussian beam, the fringe visibility follows an envelope as highlighted by the dashed line in figure $2 \mathrm{c}$ ).

The wave reflected by a FPI, due to an incident plane wave, is composed of a sum of partially reflected planes waves, of order $n \geq 0$, each of which completes $n$ double passes of the cavity. Resonance occurs when each such wave of order $n>0$ arrives at the entrance mirror mutually in phase and $\pi$ out phase with the 0 order reflected wave. The phase developed during a double pass of the cavity changes if the incidence angle changes, introducing a phase difference between each partial reflection. The phase of the $n^{\text {th }}$ order partial reflection is thus proportional to $n$. It thus follows that as higher reflectivity mirrors lead to a higher number of partial reflection resulting in visibility variations as observed in figure $2 \mathrm{c}$ ).

\section{TIME RESPONSE}

Figure 2 d) represents the time response in reflection of a FPI in both resonance and out of resonance conditions. In the second case the measured light follows the input power since there is no delay between the incident and reflected light.

The time response in the resonance case is slightly more complex due to the large number of partial reflections. It is possible to highlight three phases: first there is insufficient light within to negate the 0 order reflected wave; the second phase is the balanced case where partially reflected light inside the cavity negates the 0 order reflection; the third phase consists of the gradual release of the light stored inside the cavity.

\section{CONCLUSION}

In this paper we presented newly developed models that predict experimentally observed ITFs. Using both newly models elaborated we studied and increased our understanding of the FPI working principle and limitations.

\section{REFERENCES}

[1] E. Zhang, J. Laufer, and P. Beard, "Backward-mode multiwavelength photoacoustic scanner using a planar Fabry-Perot polymer film ultrasound sensor for high-resolution three-dimensional imaging of biological tissues," Applied Optics, vol. 47, no. 4, p. 561, 2008.

[2] H. Fujiwara, Spectroscopic Ellipsometry Principles and Applications, 1997, vol. 158 , no. 6.

[3] M. Vaughan, The Fabry-Perot interferometer: history, theory, practice and applications. Routledge, 2017. 